SLAC-PUB-8016

November 1998

\title{
LIGHT-FRONT QUANTIZED CHIRAL SCHWINGER MODEL AND ITS VACUUM STRUCTURE
}

\author{
Prem P. Srivastava \\ Stanford Linear Accelerator Center, Stanford University, Stanford, California 94309
}

\begin{abstract}
The bosonized Chiral Schwinger model (CSM) is quantized on the light-front (LF). The physical Hilbert space of CSM is obtained directly once the constraints on the LF phase space are eliminated. The discussion of the degenerate vacua and the absence in the CSM of the $\theta$-vacua, as found in the Schwinger model (SM), becomes straightforward. The differences in the structures of the the mass excitations and the vacua in these gauge theories are displayed transparently. The procedure followed is the one used successfully in the previous works for describing the spontaneous symmetry breaking (SSB) and the SM on the LF. The physical contents following from the LF quantized theory agree with those known in the conventional treatment. The LF hyperplane is argued to be equally appropriate as the conventional equaltime one for the canonical quantization. Some comments on the irrelevance, in quantized field theory, of the fact that the hyperplanes $x^{ \pm}=0$ constitute characteristic surfaces of hyperbolic partial differential equation are also made.
\end{abstract}

\footnotetext{
${ }^{1}$ Research partially supported by the Department of Energy under contract DE-AC03-76SF00515

${ }^{2}$ E-mail: prem@slac.stanford.edu or prem@lafexsu1.lafex.cbpf.br. On leave of absence from Instituto de Física, UERJ-Universidade do Estado de Rio de Janeiro, Brasil.
} 


\section{Introduction}

Dirac [1], in his paper in 1949, discussed the problem of constructing a dynamical theory of physical system which would incorporate in it the principle of quantization together with that of the special relativity theory. The LF quantization which studies the relativistic quantum dynamics on the hyperplanes : $x^{0}+x^{3} \equiv \sqrt{2} x^{+}=$const., called the front form theory, was also proposed there. The instant form or the conventional equal-time theory on the contrary uses the $x^{0}=$ const. hyperplanes. The former studies the evolution of the relativistic dynamical system in $x^{+}$while the latter in $x^{0}$. The LF coordinates $x^{\mu}:\left(x^{+}, x^{-}, x^{\perp}\right)$, where $x^{ \pm}=\left(x^{0} \pm x^{3}\right) / \sqrt{2}=x_{\mp}$ and $x^{\perp}=$ $\left(x^{1}, x^{2}\right)$, are convenient to use in the front form theory. They are not related by a Lorentz transformation to the coordinates $\left(x^{0} \equiv t, x^{1}, x^{2}, x^{3}\right)$ usually employed in the instant form theory and as such the same physical content in a dynamical theory may acquire different description in the two treatments. The discussion from the LF quantized field theory may also be of relevance towards the understanding, say, of the simultaneous inclusion in dynamical theory of the principles of the general covariance and the quantization円.

We will make the convention to regard $\mathrm{l}^{+}$as the LF-time coordinate while $x^{-} \equiv x$ as the longitudinal spatial coordinate. The (temporal) evolution in $t$ or $x^{+} \equiv \tau$ of the system is generated by Hamiltonians which are very different in the two forms of the theory.

Consider [2] the invariant distance between two spacetime points: $(x-y)^{2}=$ $\left(x^{0}-y^{0}\right)^{2}-(\vec{x}-\vec{y})^{2}=2\left(x^{+}-y^{+}\right)\left(x^{-}-y^{-}\right)-\left(x^{\perp}-y^{\perp}\right)^{2}$. On an equal $x^{0}=y^{0}=$ const. hyperplane the points have spacelike separation except for if they are coincident when it becomes lightlike one. On the LF with $x^{+}=y^{+}=$const. the distance becomes independent of $\left(x^{-}-y^{-}\right)$and the seperation is again spacelike; it becomes lightlike one when $x^{\perp}=y^{\perp}$ but with the difference that now the points need not necessarily be coincident along the longitudinal direction. The LF field theory hence need not necessarily be local in $x^{-}$, even if the corresponding instant form theory is given to be a local one in all the three spatial coordinates $\vec{x}$. For example, the commutator $\left[A\left(x^{+}, x^{-}, x^{\perp}\right), B\left(0,0,0^{\perp}\right)\right]_{x^{+}=0}$ of two scalar observables would vanish on the grounds of microcausality principle if $x^{\perp} \neq 0$ since $\left.x^{2}\right|_{x^{+}=0}$ is spacelike. Its value would be thus proportional to $\delta^{2}\left(x^{\perp}\right)$ and a finite number of its derivatives, implying locality only in $x^{\perp}$ but not necessarily so in $x^{-}$. Similar arguments in the instant form theory lead to the locality in all the three spatial coordinates. Both of the commutators

${ }^{1}$ We recall the experience with the discovery of the Kruskal-Szekers coordinates in early sixtees which shed a new light on the problem of the Schwarzshild singularity in the theory of gravitation.

2 The coordinates $x^{+}$and $x^{-}$appear in a symmetric fashion and we note that $\left[x^{+}, \frac{1}{i} \partial^{-}\right]=$ $\left[x^{-}, \frac{1}{i} \partial^{+}\right]=i$ where $\partial^{ \pm}=\partial_{\mp}=\left(\partial^{0} \pm \partial^{3}\right) / \sqrt{2}$ etc.. 
$[A(x), B(0)]_{x^{+}=0}$ and $[A(x), B(0)]_{x^{0}=0}$ are nonvanishing only on the light-cone.

We remark that in the LF quantization we time order with respect to $\tau$ rather than $t$. The microcausality principle, however, ensures that the retarded commutators $[A(x), B(0)] \theta\left(x^{0}\right)$ and $[A(x), B(0)] \theta\left(x^{+}\right)$, which appear [3] in the S-matrix elements, do not lead to disagreements in the two formulations. In the regions $x^{0}>0, x^{+}<0$ and $x^{0}<0, x^{+}>0$, where the commutators seem different the $x^{2}$ is spacelike. Hence, if we assume the microcausality principle, the LF hyperplane seems equally appropriate as the conventional one of the instant form theory for the canonical quantization.

The structure of the phase space in the front form theory is different from that of the one in the conventional theory. For example, the LF vacuum is generally found simpler [4, 5] and in many cases the interacting theory vacuum is seen to coincide with the perturbation theory one. The SSB in the scalar theory is also described (2) differently on the LF. The broken continuous symmetry is inferred now from the residual symmetry of the LF Hamiltonian operator while the symmetry of the LF vacuum remains unbroken, which is in contrast to the conventional description in which the symmetry of the vacuum state is broken while the Hamiltonian remains invariant. The expression which counts the number of Goldstone bosons in the front form theory, however, is found to be the same as in the conventional treatment. The Coleman's theorem on the absence of the Goldstone bosons in two dimensional scalar theory also finds a new demonstration [2] in the front form theory.

A recent study [6] on the LF quantized SM showed that we are led directly to the physical Hilbert space once the constraints on the phase space are eliminated. The well known [7] condensate or $\theta$-vacua and their continuum normalization were shown to emerge [6] in a straightforward fashion. In the present work we study the bosonized CSM on the LF and demonstrate in equally direct fashion its degenerate vacuum structure along with the absence of the condensate or $\theta$-vacua in this model.

An important advantge pointed out by Dirac of the front form theory is that in it seven out of the ten Poincaré generators are kinematical, e.g., they leave the hyperplane $x^{+}=0$ invariant [1]. They are [ $P^{+}, P^{1}, P^{2}, M^{12}=-J_{3}, M^{+-}=M^{03}=-K_{3}, M^{1+}=$ $\left(K_{1}+J_{2}\right) / \sqrt{2}$ and $M^{+2}=\left(K_{2}-J_{1}\right) / \sqrt{2}$. In the conventional theory only six such ones [1], viz., $\vec{P}$ and $M^{i j}=-M^{i j}$, leave the hyperplane $x^{0}=0$ invariant.

We recall also that the LF field theory was rediscovered [8] by Weinberg in his Feynman rules adapted for the infinite momentum frame. It was demonstrated [9] latter that these rules, in fact, correspond to the front form quantized theory. It was

${ }^{3}$ In the standard notation $K_{i}=-M^{0 i}, J_{i}=-(1 / 2) \epsilon_{i j k} M^{k l}, i, j, k=1,2,3$. The generator $K_{3}$ is dynamical one in the instant form theory. It is in contrast kinematical in the front form theory where it generates the scale transformations of the LF components of $x^{\mu}, P^{\mu}$ and $M^{\mu \nu}$, with $\mu, \nu=+,-, 1,2$ and where $P^{ \pm}=\left(P^{0} \pm P^{3}\right) / \sqrt{2}$ etc.. 
also successfully employed in the nonabelian bosonization of the field theory of $\mathrm{N}$ free Majorana fermions, where the corresponding LF current algebra was compared [10] with the one in the bosonized theory described by the WZNW action at the critical point.

The interest in LF quantization has been revived «, 5] also due to the difficulties encountered in the computation, in the conventional framework, of the nonperturbative effects in the context of QCD and the problem of the relativistic bound states of light fermions [5, 4] in the presence of the complex vacuum structure. The front-form dynamics may serve as a complementary tool where we have a simple vacuum while the complexity of the problem is now transferred to the LF Hamiltonian. In the case of the scalar field theory, for example, the LF Hamiltonian is in fact found [1], 2] to be nonlocal due to the presence off constraint equations in the Hamiltonian formulation.

The chiral $Q E D_{2}$ or CSM, employing the conventional framework, has received [12, 13] much attention since Jackiw and Rajaraman [14] pointed out that, despite the gauge anomaly it developed due to the renormalization ambiguity, the theory can be shown to be unitary and consistently quantized.

The procedure used [2, 11] previously for explaining the SSB on the LF and recently [6] in the bosonized SM is applied below to discuss the CSM. The scalar field is first separated, based on physical considerations, into the dynamical bosonic condensate variable $\omega\left(\tau, x^{\perp}\right)$ and the quantum fluctuation field $\varphi\left(\tau, x^{-}, x^{\perp}\right)$, e.g., $\phi\left(\tau, x^{-}, x^{\perp}\right)=$ $\omega\left(\tau, x^{\perp}\right)+\varphi\left(\tau, x^{-}, x^{\perp}\right)$. The standard Dirac method [15] is subsequently applied to construct the self-consistent LF Hamiltonian framework which is then quantized canonically. The c- or q-number nature of the condensate $\omega$ emerges from inside the theory itself.

Sec. 2 discusses how the condensate variable is subtracted out by simple field redefinition from the Lagrangian of the bosonized CSM on the LF. The canonical Hamiltonian framework is constructed in Sec. 3 following the standard Dirac method. Its quantization, the structure of the Hilbert space, the degenerate vacua and the mass spectrum are studied in Sec. 4. Conclusions are summarized in Sec. 5 where some comments are also made on the relevance to the LF quantization of the fact that the $x^{ \pm}=0$ hyperplanes are the characteristic surfaces of hyperbolic partial differential equation. In order to solve the Cauchy initial value problem in the classical theory of partial differential equations we would be required to specify the data on both of these surfaces; in the context of the LF quantization we need to select only one of the hyperplanes.

\footnotetext{
${ }^{4}$ In fact, Dirac [1] in his paper does give an example showing that the potential must be constrained if we incorporate in the dynamical theory the principles of quantization and special relativity.
} 


\section{Bosonized CSM on the LF. Absence of $\theta$-vacua}

The Lagrangian density of the chiral $Q E D_{2}$ or CSM model under consideration is

$$
\mathcal{L}=-\frac{1}{4} F^{\mu \nu} F_{\mu \nu}+\bar{\psi}_{R} i \gamma^{\mu} \partial_{\mu} \psi_{R}+\bar{\psi}_{L} \gamma^{\mu}\left(i \partial_{\mu}+2 e \sqrt{\pi} A_{\mu}\right) \psi_{L}
$$

wheref $\psi=\psi_{R}+\psi_{L}$ is a two-component spinor field and $A_{\mu}$ is the abelian gauge field The classical Lagrangian (1) is invariant under the local $U(1)$ gauge transformations $A_{\mu} \rightarrow A_{\mu}+\partial_{\mu} \alpha /(2 \sqrt{\pi} e), \psi \rightarrow\left[P_{R}+e^{i \alpha} P_{L}\right] \psi$ and under the global $U(1)_{5}$ chiral transformations $\psi \rightarrow \exp \left(i \gamma_{5} \alpha\right) \psi$.

The model under study can be solved completely using the technique of bosonization. The latter consists in the replacement of a known system of fermions with a theory of bosons which has a completely equivalent physical content, including, for example, identical spectra, the same current commutation relations and the energymomentum tensor when expressed in terms of the currents. The bosonized version of (1) is convenient to study the vacuum structure and it was shown [14 to be

$$
S=\int d^{2} x\left[-\frac{1}{4} F_{\mu \nu} F^{\mu \nu}+\frac{1}{2} \partial_{\mu} \phi \partial^{\mu} \phi+e A_{\nu}\left(\eta^{\mu \nu}-\epsilon^{\mu \nu}\right) \partial_{\mu} \phi+\frac{1}{2} a e^{2} A_{\mu} A^{\mu}\right]
$$

Here the explicit mass term for the gauge field parametrized by the constant parameter $a$ represents a regularization ambiguity and the breakdown of $U(1)$ gauge symmetry. The action (2) may be derived by the functional integral or the canonical quantization methods.

Following the procedure successfully used in the earlier works we first make the separation: $\phi\left(\tau, x^{-}\right)=\omega(\tau)+\varphi\left(\tau, x^{-}\right)$. The subsequent application of the Dirac method then enabled us to give [2, 11] the description on the LF of the SSB in the scalar theory and also the variable $\omega$ was shown there to come out as a c-number (background field). On the other hand in the bosonized SM on the LF it turned out to be q-number operator whose eigenvalues were shown [6] to label the condensate or $\theta$-vacua. We set $\int d x^{-} \varphi\left(\tau, x^{-}\right)=0$ so that the entire zero-momentum mode of $\phi$ is represented by the condensate variable and recall [6] also that the chiral transformation is defined by: $\omega \rightarrow \omega+$ const., $\varphi \rightarrow \varphi$, and $A_{\mu} \rightarrow A_{\mu}$. This ensures that the boundary conditions on the $\varphi$ are kept unaltered under such transformations and our mathematical framework may be considered well posed, before we proceed to build the canonical Hamiltonian framework.

Written explicitly (2) takes the following form on the LF

$$
S=\int d^{2} x\left[\dot{\varphi} \varphi^{\prime}+\frac{1}{2}\left(\dot{A}_{-}-A_{+}^{\prime}\right)^{2}+a e^{2}\left[A_{+}+\frac{2}{a e}(\dot{\omega}+\dot{\varphi})\right] A_{-}\right]
$$

\footnotetext{
${ }^{5}$ Here $\gamma^{0}=\sigma_{1}, \gamma^{1}=i \sigma_{2}, \gamma_{5}=-\sigma_{3}, x^{\mu}:\left(x^{+} \equiv \tau, x^{-} \equiv x\right)$ with $\sqrt{2} x^{ \pm}=\sqrt{2} x_{\mp}=\left(x^{0} \pm x^{1}\right)$, $A^{ \pm}=A_{\mp}=\left(A^{0} \pm A^{1}\right) / \sqrt{2}, \psi_{L, R}=P_{L, R} \psi, P_{L}=\left(1-\gamma_{5}\right) / 2, P_{R}=\left(1+\gamma_{5}\right) / 2, \bar{\psi}=\psi^{\dagger} \gamma^{0}$.
} 
where an overdot (a prime) indicates the partial derivative with respect to $\tau(x)$. In order to suppress the finite volume effects we work in the continuum formulation and require, based on physical considerations, that the fields satisfy the boundary conditions needed for the existence of their Fourier transforms in the spatial variable $x^{-}$. We note now that $A_{+}$appears in the action (3) as an auxiliary field, without a kinetic term. It is clear that the condensate variable may thus be subtracted out from the theory using the frequently adopted procedure of field redefinition [16] on it: $A_{+} \rightarrow A_{+}-2 \dot{\omega} /(a e)$, obtaining thereby

$$
\mathcal{L}_{C S M}=\dot{\varphi} \varphi^{\prime}+\frac{1}{2}\left(\dot{A}_{-}-A_{+}^{\prime}\right)^{2}+2 e \dot{\varphi} A_{-}+a e^{2} A_{+} A_{-},
$$

which signals the emergence of a different structure of the Hilbert space compared to that of the SM. There the condensate or $\theta$-vacua emerged due to the presence of the additional variable $\omega$ in the theory.

\section{LF Hamiltonian Framework}

The Lagrange eqs. following from (4) are

$$
\begin{aligned}
\partial_{+} \partial_{-} \varphi & =-e \partial_{+} A_{-}, \\
\partial_{+} \partial_{+} A_{-}-\partial_{+} \partial_{-} A_{+} & =a e^{2} A_{+}+2 e \partial_{+} \varphi \\
\partial_{-} \partial_{-} A_{+}-\partial_{+} \partial_{-} A_{-} & =a e^{2} A_{-} .
\end{aligned}
$$

and for $a \neq 1$ they lead to:

$$
\begin{array}{r}
\square G(\tau, x)=0 \\
{\left[\square+\frac{e^{2} a^{2}}{(a-1)}\right] E(\tau, x)=0,}
\end{array}
$$

where $E=\left(\partial_{+} A_{-}-\partial_{-} A_{+}\right)$and $G=(E-a e \varphi)$. Both the massive and massless scalar excitations are present in the theory and the tachyons would be absent in the specrtum if $a>1$; the case considered in this paper. We will confirm in the Hamiltonian framework below that the $E$ and $G$ represent, in fact, the two independent field operators on the LF phase space.

The Dirac procedure [15] as applied to (4) is straightforward. The canonical momenta are $\pi^{+} \approx 0, \pi^{-} \equiv E=\dot{A}_{-}-A_{+}^{\prime}, \pi_{\varphi}=\varphi^{\prime}+2 e A_{-}$which result in two primary

\footnotetext{
${ }^{6}$ In the SM we have $[6]: L=\int d x^{-}\left[\dot{\varphi} \varphi^{\prime}+\frac{1}{2}\left(\dot{A}_{-}-A_{+}^{\prime}\right)^{2}-(e / \sqrt{\pi})\left(A_{+} \varphi^{\prime}-A_{-} \dot{\varphi}\right)\right]+(e / \sqrt{\pi}) \dot{\omega} h(\tau)$
} where $h(\tau)=\int d x^{-} A_{-}\left(\tau, x^{-}\right)$. 
weak constraints $\pi^{+} \approx 0$ and $\Omega_{1} \equiv\left(\pi_{\varphi}-\varphi^{\prime}-2 e A_{-}\right) \approx 0$. A secondary constraint $\Omega_{2} \equiv \partial_{-} E+a e^{2} A_{-} \approx 0$ is shown to emerge when we require the $\tau$ independence (persistency) of $\pi^{+} \approx 0$ employing the preliminary Hamiltonian

$$
H^{\prime}=H_{c}^{l f}+\int d x u_{+} \pi^{+}+\int d x u_{1} \Omega_{1},
$$

where $u_{+}$and $u_{1}$ are the Lagrange multiplier fields and $H_{c}^{l f}$ is the canonical Hamiltonian

$$
H_{c}^{l f}=\int d x\left[\frac{1}{2} E^{2}+E A_{+}^{\prime}-a e^{2} A_{+} A_{-}\right] .
$$

and we assume initially the standard equal- $\tau$ Poisson brackets : $\left\{E^{\mu}\left(\tau, x^{-}\right), A_{\nu}\left(\tau, y^{-}\right)\right\}=-\delta_{\nu}^{\mu} \delta\left(x^{-}-y^{-}\right),\left\{\pi_{\varphi}\left(\tau, x^{-}\right), \varphi\left(\tau, y^{-}\right)\right\}=-\delta\left(x^{-}-y^{-}\right)$etc.. The persistency requirement for $\Omega_{1}$ results in an equation for determining $u_{1}$. The procedure is repeated with the following extended Hamiltonian which includes in it also the secondary constraint

$$
H_{e}^{l f}=H_{c}^{l f}+\int d x u_{+} \pi^{+}+\int d x u_{1} \Omega_{1}+\int d x u_{2} \Omega_{2} .
$$

No more secondary constraints are seen to arise; we are left with the persistency conditions which determine the multiplier fields $u_{1}$ and $u_{2}$ while $u_{+}$remains undetermined. We also find] $(C)_{i j}=\left\{\Omega_{i}, \Omega_{j}\right\}=D_{i j}\left(-2 \partial_{x} \delta(x-y)\right)$ where $i, j=1,2$ and $D_{11}=1, D_{22}=a e^{2}, D_{12}=D_{21}=-e$ and that $\pi^{+}$has vanishing brackets with $\Omega_{1,2}$. The $\pi^{+} \approx 0$ is first class weak constraint while $\Omega_{1}$ and $\Omega_{2}$, which does not depend on $A_{+}$or $\pi^{+}$, are second class ones.

We go over from the Poisson bracket to the Dirac bracket $\{,\}_{D}$ constructed in relation to the pair, $\Omega_{1} \approx 0$ and $\Omega_{2} \approx 0$

$$
\{f(x), g(y)\}_{D}=\{f(x), g(y)\}-\iint d u d v\left\{f(x), \Omega_{i}(u)\right\}\left(C^{-1}(u, v)\right)_{i j}\left\{\Omega_{j}(v), g(y)\right\} .
$$

Here $C^{-1}$ is the inverse of $C$ and we find $\left(C^{-1}(x, y)\right)_{i j}=B_{i j} K(x, y)$ with $B_{11}=$ $a /(a-1), B_{22}=1 /\left[(a-1) e^{2}\right], \quad B_{12}=B_{21}=1 /[(a-1) e]$, and $K(x, y)=$ $-\epsilon(x-y) / 4$. Some of the Dirac brackets are $\{\varphi, \varphi\}_{D}=B_{11} K(x, y) ;\{\varphi, E\}_{D}=$ $e B_{11} K(x, y) ;\{E, E\}_{D}=a e^{2} B_{11} K(x, y) ;\left\{\varphi, A_{-}\right\}_{D}=-B_{12} \delta(x-y) / 2 ;\left\{A_{-}, E\right\}_{D}=$ $B_{11} \delta(x-y) / 2 ;\left\{A_{-}, A_{-}\right\}_{D}=B_{12} \partial_{x} \delta(x-y) / 2$ and the only nonvanishing one involving $A_{+}$or $\pi^{+}$is $\left\{A_{+}, \pi^{+}\right\}_{D}=\delta(x-y)$.

The eqns. of motion employ now the Dirac brackets and inside them, in view of their very construction [15], we may set $\Omega_{1}=0$ and $\Omega_{2}=0$ as strong relations. The

7 We make the convention that the first variable in an equal- $\tau$ bracket refers to the longitudinal coordinate $x^{-} \equiv x$ while the second one to $y^{-} \equiv y$ while $\tau$ is suppressed. 
Hamiltonian is therefore effectively given by $H_{e}$ with the terms involving the multipliers $u_{1}$ and $u_{2}$ dropped. The multiplier $u_{+}$is not determined since the constraint $\pi^{+} \approx 0$ continues to be first class even when the above Dirac bracket is employed. The variables $\pi_{\varphi}$ and $A_{-}$are then removed from the theory leaving behind $\varphi, E, A_{+}$, and $\pi^{+}$as the remaining independent variables. The canonical Hamiltonian density reduces to $\mathcal{H}_{c}^{l f}=E^{2} / 2+\partial_{-}\left(A_{+} E\right)$ while $\dot{A}_{+}=\left\{A_{+}, H_{e}^{l f}\right\}_{D}=u_{+}$. The surface term in the canonical LF Hamiltonian may be ignored if, say, $E\left(=F_{+-}\right)$vanishes at infinity. The variables $\pi^{+}$and $A_{+}$are then seen to describe a decoupled (from $\varphi$ and $E$ ) free theory and we may hence drop these variables as well. The effective LF Hamiltonian thus takes the simple form

$$
H_{C S M}^{l f}=\frac{1}{2} \int d x E^{2}
$$

which is to be contrasted with the one found in the conventional treatment [13, 12]. $E$ and $G$ (or $E$ and $\varphi$ ) are now the independent variables on the phase space and the eqs. (6) are verified to be recovered for them which assures us of the selfconsistency [15]. We stress that in our discussion we do not employ any gauge-fixing. The same result (11) could be alternatively obtained $\rrbracket^{\Uparrow}$, however, if we did introduce the gaugefixing constraint $A_{+} \approx 0$ and made further modification on $\{,\}_{D}$ in order to implement $A_{+} \approx 0, \pi^{+} \approx 0$ as well. That it is accessible on the phase space to take care of the remaining first class constraint, but not in the Lagrangian in (4), follows from the Hamiltons eqns. of motion. We recall [6] that in the $\operatorname{SM} \varphi, \omega$, and $\pi_{\omega}=(e / \sqrt{\pi}) \int d x A_{-}$ were shown to be the independent operators and that the matter field $\varphi$ appeared instead in the LF Hamiltonian.

\section{Quantization. Vacuum structure in CSM}

The canonical quantization is peformed via the correspondence $i\{f, g\}_{D} \rightarrow[f, g]$ and we find the following equal- $\tau$ commutators

$$
\begin{aligned}
& {[E(x), E(y)]=i K(x, y) a^{2} e^{2} /(a-1),} \\
& {[G(x), E(y)]=0} \\
& {[G(x), G(y)]=i a^{2} e^{2} K(x, y) .}
\end{aligned}
$$

For $a>1$, when the tachyons are absent as seen from (6), these commutators are also physical and the independent field operators $E$ and $G$ generate the Hilbert space with a tensor product structure of the Fock spaces $F_{E}$ and $F_{G}$ of these fields with the positive definite metric.

\footnotetext{
${ }^{8}$ A similar discussion is encountered also in the LF quantized Chern-Simons-Higgs system [17].
} 
We can make, in view of (12), the following LF momentum space expansions

$$
\begin{aligned}
& E(x, \tau)=\frac{a e}{\sqrt{(a-1)} \sqrt{2 \pi}} \int_{-\infty}^{\infty} d k \frac{\theta(k)}{\sqrt{2 k}}\left[d(k, \tau) e^{-i k x}+d^{\dagger}(k, \tau) e^{i k x}\right], \\
& G(x, \tau)=\frac{a e}{\sqrt{2 \pi}} \int_{-\infty}^{\infty} d k \frac{\theta(k)}{\sqrt{2 k}}\left[g(k, \tau) e^{-i k x}+g^{\dagger}(k, \tau) e^{i k x}\right]
\end{aligned}
$$

where the operators $\left(d, g, d^{\dagger}, g^{\dagger}\right)$ satisfy the canonical commutation relations of two independent harmonic oscillators; the well known set of Schwinger's bosonic oscillators, often employed in the angular momentum theory. The expression for the Hamiltonian becomes

$$
H_{C S M}^{l f}=\delta(0) \frac{a^{2} e^{2}}{2(a-1)} \int_{k>0}^{\infty} \frac{d k}{2 k} N_{d}(k, \tau)
$$

where we have dropped the infinite zero-point energy term and note that [3] $\left[d^{\dagger}(k, \tau), d(l, \tau)\right]=-\delta(k-l), d^{\dagger}(k, \tau) d(k, \tau)=\delta(0) N_{d}(k, \tau)$ etc. with similar expressions for the independent g-oscillators. We verify that $\left[N_{d}(k, \tau), N_{d}(l, \tau)\right]=0$, $\left[N_{d}(k, \tau), N_{g}(l, \tau)\right]=0,\left[N_{d}(k, \tau), d^{\dagger}(k, \tau)\right]=d^{\dagger}(k, \tau)$ etc..

The Fock space can hence be built on a basis of eigenstates of the hermitian number operators $N_{d}$ and $N_{g}$. The ground state of CSM is degenerate and described by $\mid 0>=$ $\mid E=0) \otimes \mid G\}$ and it carries vanishing LF energy. For a fixed $k$ these states, $\mid E=$ $\left.0) \otimes\left(g^{\dagger}(k, \tau)^{n} / \sqrt{n !}\right) \mid 0\right\}$, are labelled by the integers $n=0,1,2, \cdots$. The $\theta$-vacua are absent in the CSM, however, we recall [6] that in the SM the degenerate chiral vacua are also labelled by such integers. We remark also that on the LF we work in the Minkowski space and that in our discussion we do not make use of the Euclidean space theory action, where the (classical) vacuum configurations of the Euclidean theory gauge field, belonging to the distinct topological sectors, are useful, for example, in the functional integral quantization of the gauge theory.

\section{Conclusions}

The LF hyperplane is argued to be equally appropriate as the conventional one for quantizing field theory. The discussion given above in the front form formulation is seen again to be quite transparent and the physical contents following from the quantized theory agree with those known in the conventional instant form treatment. Evidently, they should not depend on whether we employ the conventional or the LF coordinates to span the Minkowski space and study the temporal evolution of the quantum dynamical system in $t$ or $\tau$ respectively.

We note that in our context the (LF) hyperplanes $x^{ \pm}=0$ define the characteristic surfaces of hyperbolic partial differential equation. It is known from their mathematical 
theory [18] that a solution exists if we specify the initial data on both of the hyperplanes. From the present discussion and the earlier works [2, 6] we conclude that it is sufficient in the front form treatment to choose one of the hyperplanes, as proposed by Dirac [1], for canonically quantizing the theory. The equal- $\tau$ commutators of the field operators, at a fixed initial LF-time, form now a part of the initial data instead and we deal with operator differential equations. The information on the commutators on the other characteristic hyperplane seems to be already contained [6] in the quantized theory and need not be specified separately. As a side comment, the well accepted notion that a classical model field theory must be upgraded first through quantization, before we confront it with the experimental data, finds here in a sense a theoretical confirmation.

The physical Hilbert space is obtained in a direct fashion in the LF quantized CSM and SM gauge theories, once the constraints are eliminated and the appreciably reduced set of independent operators on the LF phase space identified. The CSM has in it both the massive and the massless scalar excitations while only the massive one appears in the SM. There are no condensate or $\theta$ vacua in CSM but they both have degenerate vacuum structure. In the conventional treatment [7] an extended phase space is employed and suitable constraints are required to be imposed in order to define the physical Hilbert space which would then lead to the description of the physical vacuum state. The existence of one more kinematical generator on the LF and the inherent symmetry in $x^{ \pm}$in the quantized theory seem to introduce already sufficient number of constraints in the theory leading to great deal of simplifications. Many of the ingredients like, for example, the continuuum normalization of the $\theta$-vacua in SM, which needs to be imposed in the conventional treatment are already to be found in the front form quantized theory. The functional integral method together with the LF quantization may be an efficient tool for handling the nonperturbative calculations.

A discussion parallel to the one given here can also be made in the front form theory of the gauge invariant formulation [12] of the CSM. In an earlier work [19], where the BRST-BFV functional integral quantization was employed, it was demonstrated that this formulation and the gauge noninvariant one in (2) in fact lead to the same effective action. Also the BRS-BFT quantization method proposed [20] recently can be extended to the front form theory. It was applied [21] to the action (2) and different equivalent effective actions obtained for the CSM.

\section{Acknowledgements}

The author would like to thank Werner Israel, Silvio Sorella, James Vary, Vitor Oguri, Sebastião Dias and Francisco Caruso for constructive remarks. The hospitality offered at the SLAC Theory Group and the financial aid from the CNPq, Brasil, for his participation at the 8th Workshop on Light-Cone QCD, Lutsen, MN, are gratefully acknowledged. 


\section{References}

[1] P.A.M. Dirac, Rev. Mod. Phys. 21 (1949) 392.

[2] P.P. Srivastava, Lightfront quantization of field theory in Topics in Theoretical Physics, Festschrift for Paulo Leal Ferreira, eds., V.C. Aguilera-Navarro et. al., pgs. 206-217, IFT-São Paulo, SP, Brasil (1995); hep-th/9610044 and 9312064; Nuovo Cimento A107 (1994) 549; A108 (1995) 35.

[3] see for example S.S. Schweber, Relativistic Quantum Field Theory, Row, Peterson and Co., New York, 1961; L.H. Ryder, Quantum Field Theory, Cambridge University Press, 2nd Edition, 1996.

[4] S.J. Brodsky, Light-Cone Quantized QCD and Novel Hadron Phenomenology, SLAC-PUB-7645, 1997; S.J. Brodsky and H.C. Pauli, Light-Cone Quantization and QCD, Lecture Notes in Physics, vol. 396, eds., H. Mitter et. al., SpringerVerlag, Berlin, 1991.

[5] K.G. Wilson et. al., Phys. Rev. D49 (1994) 6720; R.J. Perry, A. Harindranath, and K.G. Wilson, Phys. Rev. Lett. 65 (1990) 2959; K.G. Wilson, Nucl. Phys. B (proc. Suppl.) 17 (1990)

[6] P.P. Srivastava, Mod. Phys. Letts. A13 (1998) 1223. See also Geometry, Topology and Physics, pgs. 260-275, Eds. Apanasov et. al., Walter de Gruyter \& Co., Berlin, New York, 1997; hep-th/9610149.

[7] J. Lowenstein and J. Swieca, Ann. Physics (N.Y.) 68 (1971) 172.

[8] S. Weinberg, Phys. Rev. 150 (1966) 1313.

[9] J.B. Kogut and D.E. Soper, Phys. Rev. D1 (1970) 2901.

[10] E. Witten, Commun. Math. Phys. 92 (1984) 455; Nucl. Phys. B223 (1983) 422.

[11] P.P. Srivastava, LF quantization and SSB in Hadron Physics 94, pgs. 253-260, Eds. V. Herscovitz et. al., World Scientific, Singapore, 1995; hep-th/9412204,9412205.

[12] See for example, E. Abdalla, M.C. Abdalla and K. Rothe, Non-Perturbative Methods in Two Dimensional Quantum Field Theory, World Scientific, Singapore, 1991.

[13] See for example, D. Boyanovsky, Nucl. Phys. B294 (1987) 223; A. Bassetto, L. Griguolo and P. Zanca, Phys. Rev. D50 (1994) 1077; ref. [12].

[14] R. Jackiw and R. Rajaraman, Phys. Rev. Lett. 54 (1985) 1219; 54 (1985) 2060(E). 
[15] P.A.M. Dirac, Lectures in Quantum Mechanics, Benjamin, New York, 1964.

[16] P. van Nieuwenhuizen, Phys. Rep. 68 (1981) 189.

[17] P.P. Srivastava, Europhys. Lett. 33 (1996) 423; LF dynamics of Chern-Simons systems, ICTP, Trieste preprint IC/94/305; hep-th/9412239.

[18] See for example, I.N. Sneddon, Elements of Partial Differential Equations, McGraw-Hill, NY, 1957, pg. 111-115

[19] P.P. Srivastava, Phys. Lett. B235 (1990) 287.

[20] I.A. Batalin and I.V. Tyutin, Int. J. Mod. Phys. A6 (1991) 3255.

[21] P.P. Srivastava, BRS-BFT quantization of the CSM on the light-front, paper LP002, Session P17, Intl. Symp. on Lepton-Photon Interactions- LP'97, July 1997, Hamburg. Available as .ps file on the DESY database. 